\title{
Características cefalométricas do Padrão Face Longa: considerando o dimorfismo sexual*
}

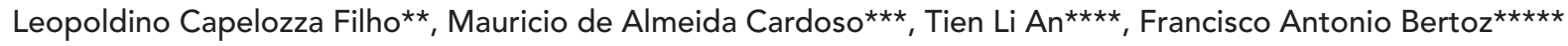

\begin{abstract}
Resumo
Objetivo: o presente estudo avaliou a hipótese de variação das características cefalométricas de acordo com o gênero para portadores de Padrão Face Longa. Metodologia: foi analisado um total de 73 telerradiografias em norma lateral, sendo 34 Padrão Face Longa e 39 Padrão I (grupo controle), selecionadas com base na morfologia facial, sem considerar as relações oclusais. Foram avaliados: padrão de crescimento facial, alturas faciais anteriores e posterior, relação maxilomandibular, além das relações dentárias com suas bases apicais. Resultados: o grupo controle (Padrão I) apresentou dimorfismo ( $\mathrm{p}<0,001)$ no comprimento efetivo da maxila e da mandíbula, além das alturas faciais avaliadas. Foram significantes também $(\mathrm{p}<0,05)$ as proporções entre as alturas faciais e as distâncias entre os incisivos e molares em relação aos planos palatino e mandibular. Para os portadores de Padrão Face Longa, as mesmas variáveis cefalométricas não mostraram diferenças significantes entre os gêneros ( $p>0,05)$, exceto os comprimentos efetivos da maxila e da mandíbula, além da altura facial anterior inferior e posterior $(\mathrm{p}<0,05)$. Conclusões: concluiu-se que enquanto ocorreu dimorfismo nos indivíduos Padrão I, para as estruturas influenciadas pela diferença no tamanho esquelético entre os gêneros, isso não aconteceu nos portadores de Padrão Face Longa. Essa maior similaridade entre os gêneros provavelmente ocorreu porque a deformidade, mais grave no gênero feminino, foi suficiente para anular o menor tamanho esquelético esperado para estes indivíduos.
\end{abstract}

Palavras-chave: Cefalometria. Deformidades. Dimorfismo. Padrão Face Longa.

\section{INTRODUÇÃO}

O Padrão Face Longa é uma deformidade de prevalência relativamente baixa, em torno de $1,5 \%$ da população mundial ${ }^{18}$. O grupo de indivíduos portadores é caracterizado por grandes desvios morfológicos em relação ao Padrão $I^{11,12}$, demonstrando significativo impacto estético. Para os indivíduos com face desagradável, um procedimento ortodôntico-cirúrgico está indica$\mathrm{do}^{24,39,41,42}$.

Resumo da Dissertação apresentada à Faculdade de Odontologia do "Campus de Araçatuba-UNESP", para obtenção do grau de Mestre em Odontologia (Área de Concentração: Ortodontia) pelo autor Mauricio de Almeida Cardoso.

** Professor Doutor da Faculdade de Odontologia da Universidade de São Paulo, USP-Bauru e membro do Setor de Ortodontia do HRAC da Universidade de São Paulo, USP-Bauru. Professor do Programa de Pós-graduação em Ortodontia da Faculdade de Odontologia de Araçatuba - UNESP.

*** Mestre e Doutorando em Ortodontia pela Universidade Estadual Paulista, UNESP-Araçatuba. Professor em nível de Graduação e Pós-Graduação da Universidade do Sagrado Coração, USC-Bauru.

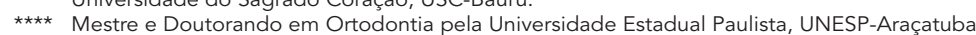

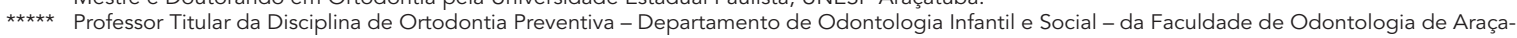
tuba - UNESP. Professor do Programa de Pós-graduação em Ortodontia da faculdade de Odontologia de Araçatuba - UNESP. 
A principal queixa desses pacientes é o excesso de exposição dentária ântero-superior com os lábios em repouso e dentogengival durante o sorriso $^{2}$, ocasionado pelo aumento excessivo do terço inferior da face f $^{2,15,35,37,42}$.

A literatura é unânime quanto às características faciais e cefalométricas desses pacientes $^{2,5,6,14,15,17,18,19,20,21,23,25,26,27,30,34,35,36,37,38,42}$, inadequadamente denominados portadores de mordida aberta esquelética ${ }^{3}$ ou síndrome da face longa ${ }^{8,31,32}$, ao invés de nominá-los pelo erro esquelético ${ }^{11,12}$.

As amostras utilizadas nos estudos de Vig, Turvey ${ }^{41}$; Haralabakis et al. ${ }^{21}$ e Cardoso et al. ${ }^{14}$ parecem mostrar uma predominância desta deformidade para o gênero feminino. Essa provável falsa impressão casuística pode ser atribuída a uma maior procura do tratamento por representantes femininos, em decorrência do prejuízo na estética facial.

Há poucos dados na literatura sobre dimorfismo sexual para características cefalométricas dos indivíduos Padrão Face Longa. Eles sugerem a hipótese de diferença entre os gêneros feminino e masculino $21,23,25$, justificando assim o presente estudo, com objetivo de avaliar e dar ou não subsídio a esta hipótese.

\section{MATERIAL E MÉTODOS \\ Material}

O presente estudo foi devidamente aprovado pelo Comitê de Ética de Pesquisa em Humanos da Universidade Estadual Paulista, Campus de Araçatuba (FOA 2001/0810). Foram selecionadas, a partir da documentação existente no Centro de Cirurgia e Ortodontia, 34 telerradiografias em norma lateral, na fase pré-tratamento, de indivíduos Padrão Face Longa, masculinos ( $\mathrm{n}=12$ ) e femininos ( $\mathrm{n}=22)$, brasileiros, brancos, com indicação para preparo ortodôntico e cirurgia ortognática na maxila, mandíbula e mento ${ }^{13}$ (Tab. 1). Como critério de inclusão, nenhum indivíduo havia sido submetido a tratamento ortopédico e/ou ortodôntico prévio, nem apresentava sindromes e/ou assimetrias significativas. Todos os indivíduos selecionados apresentavam idade acima de 15 anos, numa média de 22 anos e 2 meses ( \pm 7 anos e 5 meses) para a amostra total, sendo 25 anos e 4 meses ( \pm 7 anos e 4 meses) e 16 anos e 3 meses ( \pm 1 ano e 3 meses) as idades médias para os gêneros feminino e masculino, respectivamente. Deve-se ressaltar que a seleção da amostra dos indivíduos Padrão Face Longa foi baseada unicamente na morfologia facial, independente das relações sagitais dos primeiros molares permanentes, de acordo com a proposta diagnóstica de Capelozza Filho em 2004 ${ }^{11}$.

Considerou-se, na avaliação da fotografia do perfil facial, as seguintes características: 1) terço inferior da face aumentado em relação ao terço médio; 2) linha queixo-pescoço reduzida e ângulo queixo-pescoço fechado; 3) ausência de selamento labial passivo e 4) excesso de exposição dos incisivos superiores com os lábios em repouso (Fig. 1).

Os requisitos técnicos para seleção das telerradiografias cefalométricas laterais foram a boa qualidade quanto à nitidez, posicionamento adequado da cabeça no cefalostato, contraste das estruturas ósseas e de tecido mole, além de uma adequada condição de visualização dos ápices dos incisivos e molares superiores e inferiores.

Para o grupo controle, utilizaram-se as telerradiografias de 39 indivíduos Padrão $\mathrm{I}^{11}$ (26 feminino e 13 masculino), brancos, brasileiros, adultos, pertencentes de uma parte da amostra de 100 indivíduos do estudo realizado por Reis et al. ${ }^{33}$ (Tab. 1). A idade média foi de 22 anos e 10 meses $( \pm$ 2 anos e 10 meses), sendo 19 anos e 7 meses ( \pm 3 anos e 1 mês) e 22 anos e 8 meses ( \pm 2 anos e 5 meses) as idades médias dos indivíduos, femininos e masculinos, respectivamente. Os indivíduos controle caracterizaram-se por apresentar: 1) selamento labial passivo; 2) perfil levemente convexo; 3) terços faciais proporcionais; 4) linha queixo-pescoço paralela ao plano de Camper; 5) sulco mentolabial normal, com igual participação do mento e do lábio e 6) sem nenhum tratamento ortopédico e ortodôntico prévio. 

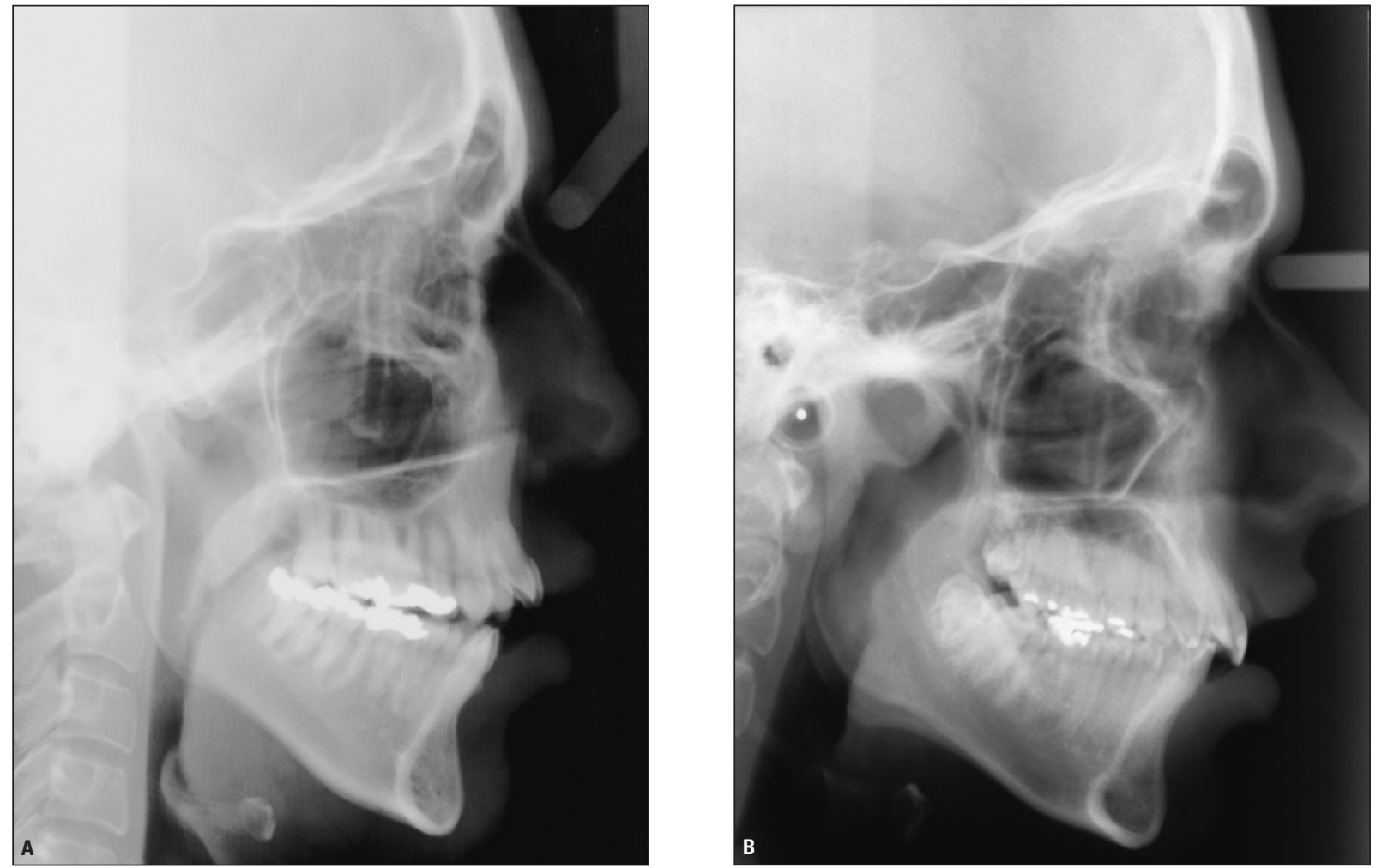

FIGURAS 1 - Radiografias cefalométricas do grupo Padrão Face Longa, ilustrando a maior gravidade da doença no gênero feminino (A) em relação ao gênero masculino (B).

Tabela 1 - Freqüência dos gêneros feminino e masculino dos grupos Padrão I e Padrão Face Longa.

\begin{tabular}{cccc} 
padrão & gênero feminino & gênero masculino & total \\
\hline $\begin{array}{c}\text { grupo Padrão I } \\
\text { grupo Padrão } \\
\text { Face Longa }\end{array}$ & $\mathrm{n}=26$ & $\mathrm{n}=13$ & $\mathrm{n}=39$ \\
total & $\mathrm{n}=48$ & $\mathrm{n}=12$ & $\mathrm{n}=34$ \\
& $\mathrm{n}=25$ & $\mathrm{n}=73$ \\
\hline
\end{tabular}

\section{Método}

As telerradiografias cefalométricas laterais foram digitalizadas por meio de scanner, sendo as imagens submetidas à análise do programa Radiocef 2.0, seguindo as normas especificadas pelo fabricante ${ }^{40}$.
Para a obtenção das medidas lineares, angulares e de proporção, a demarcação dos pontos previamente estabelecidos foi realizada nas imagens escaneadas das telerradiografias cefalométricas laterais, por um único profissional. Os resultados foram armazenados e posteriormente submetidos à avaliação estatística.

\section{Grandezas}

O método adotado neste estudo já foi previamente utilizado nos trabalhos de Cardoso ${ }^{13}$, Reis et al. ${ }^{33}$ e Cardoso et al. ${ }^{14}$ Foram utilizadas as seguintes grandezas:

- Lineares: Co-Gn; Co-A; DifMxMd; AFAT; AFAI; AFAM; AFP; AFATperp (altura facial anterior total perpendicular); AFAIperp (altura facial anterior inferior perpendicular); 1 -PP; $\underline{6}-\mathrm{PP}$; 1-PM e 6-PM.

- Angulares: SNA; SNB; ANB; AnGon (Ângulo 
goníaco); AnPP (Ângulo do plano palatino); 1.PP; IMPA; Al/3IF (Ângulo do terço inferior da face); AnPM (Ângulo do plano mandibular) e NAP.

- Proporções: AFAIperp/AFATperp; AFAI/ AFAT; AFAM/AFAI e AFP/AFAT.

\section{Análise estatística}

Para todas as variáveis mensuradas foram calculados médias e desvios-padrão, bem como os valores máximos e mínimos. Com o objetivo de detectar as diferenças entre os grupos estudados, utilizou-se o teste $t$ para dados independentes, ao nível de 5\% de significância. No presente estudo, realizaram-se quatro tipos de comparações: 1) entre indivíduos dos gêneros feminino e masculino Padrão I; 2) entre indivíduos dos gêneros feminino e masculino Padrão Face Longa; 3) entre indivídu- os Padrão I e Padrão Face Longa do gênero feminino e 4) entre indivíduos Padrão I e Padrão Face Longa do gênero masculino.

Para avaliar o erro do método, foram selecionadas aleatoriamente 22 telerradiografias que foram novamente avaliadas por meio do programa Radiocef 2.0. Foram comparadas os valores das duas mensurações por meio do teste $t$ para o cálculo do erro sistemático. Utilizou-se a fórmula $\mathrm{S}^{2}=\Sigma \mathrm{d}^{2} / 2 \mathrm{n}$, preconizada por Dahlberg, para verificar o erro aleatório. Pela fórmula, $\mathrm{S}$ é o valor do erro, d é a diferença entre as duas mensurações e n é a quantidade de repetições realizadas ${ }^{1,22}$. De acordo com a estimativa do erro do método, não foram detectadas diferenças estatisticamente significantes quanto ao erro sistemático, enquanto os valores do erro aleatório obtidos encontravam-se dentro de um limite tolerável (Tab. 2).

Tabela 2 - Erro sistemático e erro casual (Dahlberg).

\begin{tabular}{|c|c|c|c|c|c|c|c|}
\hline \multirow[b]{2}{*}{ variáveis } & \multicolumn{2}{|c|}{$1^{\mathrm{a}}$ mensuração } & \multicolumn{2}{|c|}{$2^{\mathrm{a}}$ mensuração } & \multirow[b]{2}{*}{$\mathbf{t}$} & \multirow[b]{2}{*}{ p } & \multirow[b]{2}{*}{ Dahlberg } \\
\hline & $\overline{\mathbf{x}}$ & d.p. & $\overline{\mathbf{x}}$ & d.p. & & & \\
\hline Co-Gn & $125,23 \mathrm{~mm}$ & $8,78 \mathrm{~mm}$ & $124,99 \mathrm{~mm}$ & $8,69 \mathrm{~mm}$ & 1,065 & 0,299 & $0,747 \mathrm{~mm}$ \\
\hline Co-A & $94,96 \mathrm{~mm}$ & $6,78 \mathrm{~mm}$ & $94,9 \mathrm{~mm}$ & $7,15 \mathrm{~mm}$ & 0,207 & 0,838 & $1,008 \mathrm{~mm}$ \\
\hline AFAT & $130,91 \mathrm{~mm}$ & $10,1 \mathrm{~mm}$ & $130,87 \mathrm{~mm}$ & $10,1 \mathrm{~mm}$ & 0,149 & 0,883 & $0,808 \mathrm{~mm}$ \\
\hline AFAI & $76,78 \mathrm{~mm}$ & $8,01 \mathrm{~mm}$ & $76,62 \mathrm{~mm}$ & $8,23 \mathrm{~mm}$ & 0,758 & 0,457 & $0,714 \mathrm{~mm}$ \\
\hline AFAM & $56,96 \mathrm{~mm}$ & $4,08 \mathrm{~mm}$ & $56,95 \mathrm{~mm}$ & $3,8 \mathrm{~mm}$ & 0,052 & 0,959 & $0,666 \mathrm{~mm}$ \\
\hline AFP & $62,74 \mathrm{~mm}$ & $6,17 \mathrm{~mm}$ & $62,78 \mathrm{~mm}$ & $5,56 \mathrm{~mm}$ & 0,073 & 0,943 & $1,822 \mathrm{~mm}$ \\
\hline AFATperp & $130,18 \mathrm{~mm}$ & $9,7 \mathrm{~mm}$ & $130,08 \mathrm{~mm}$ & $9,68 \mathrm{~mm}$ & 0,4 & 0,693 & $0,833 \mathrm{~mm}$ \\
\hline AFAlperp & $73,92 \mathrm{~mm}$ & $6,77 \mathrm{~mm}$ & $73,71 \mathrm{~mm}$ & $6,91 \mathrm{~mm}$ & 0,906 & 0,375 & $0,797 \mathrm{~mm}$ \\
\hline $1-P P$ & $8,42 \mathrm{~mm}$ & $3,31 \mathrm{~mm}$ & $8,37 \mathrm{~mm}$ & $3,34 \mathrm{~mm}$ & 0,174 & 0,864 & $0,954 \mathrm{~mm}$ \\
\hline$\underline{6}-P P$ & $7,68 \mathrm{~mm}$ & $2,96 \mathrm{~mm}$ & $7,71 \mathrm{~mm}$ & $2,8 \mathrm{~mm}$ & 0,161 & 0,874 & $0,684 \mathrm{~mm}$ \\
\hline 1-PM & $20,48 \mathrm{~mm}$ & $3,14 \mathrm{~mm}$ & $20,81 \mathrm{~mm}$ & $3,3 \mathrm{~mm}$ & 0,727 & 0,475 & $1,547 \mathrm{~mm}$ \\
\hline 6-PM & $12,52 \mathrm{~mm}$ & $4,03 \mathrm{~mm}$ & $12,73 \mathrm{~mm}$ & $3,59 \mathrm{~mm}$ & 0,575 & 0,571 & $1,224 \mathrm{~mm}$ \\
\hline SNA & $81,1^{\circ}$ & $3,7^{\circ}$ & $81,25^{\circ}$ & $3,47^{\circ}$ & 0,537 & 0,597 & $0,947^{\circ}$ \\
\hline SNB & $76,49^{\circ}$ & $4,93^{\circ}$ & $76,68^{\circ}$ & $4,94^{\circ}$ & 1,039 & 0,31 & $0,641^{\circ}$ \\
\hline AnGon & $125,59^{\circ}$ & $5,78^{\circ}$ & $125,75^{\circ}$ & $5,37^{\circ}$ & 0,461 & 0,649 & $1,114^{\circ}$ \\
\hline AnPM & $35,81^{\circ}$ & $8,69^{\circ}$ & $35,61^{\circ}$ & $8,69^{\circ}$ & 0,717 & 0,481 & $0,941^{\circ}$ \\
\hline AnPP & $9,11^{\circ}$ & $3,47^{\circ}$ & $9,09^{\circ}$ & $3,58^{\circ}$ & 0,081 & 0,936 & $0,879^{\circ}$ \\
\hline $1 . P P$ & $112,74^{\circ}$ & $7,8^{\circ}$ & $112,43^{\circ}$ & $8,15^{\circ}$ & 0,575 & 0,572 & $1,819^{\circ}$ \\
\hline IMPA & $92,11^{\circ}$ & $7,1^{\circ}$ & $92,76^{\circ}$ & $7,02^{\circ}$ & 1,022 & 0,318 & $1,809^{\circ}$ \\
\hline $\mathrm{A} 1 / 3 \mathrm{IF}$ & $76,31^{\circ}$ & $5,47^{\circ}$ & $76,17^{\circ}$ & $5,25^{\circ}$ & 0,366 & 0,718 & $1,316^{\circ}$ \\
\hline NAP & $8,45^{\circ}$ & $6,84^{\circ}$ & $8,09^{\circ}$ & $6,8^{\circ}$ & 1,32 & 0,201 & $0,93^{\circ}$ \\
\hline
\end{tabular}




\section{RESULTADOS}

Os resultados referentes às médias, os desvios-padrão, o valor do t e a significância estatística encontrados estão ordenados nas tabelas 3 a 6 . Em síntese, foram observadas diferenças significantes entre os indivíduos dos gêneros feminino e masculino Padrão I com relação às grandezas lineares e similaridade entre as grandezas angulares (Tab. 3). Por outro lado, este quadro foi diferente para os indivíduos dos gêneros feminino e masculino Padrão Face Longa, onde houve predominância de similaridades tanto para grandezas lineares como angulares (Tab. 4). Quando indivíduos do gênero feminino Padrão I e Padrão Face Longa foram comparados, evidenciou-se diferenças significantes para a maioria das grandezas, sejam lineares ou angulares (Tab. 5). Diferentemente desses comportamentos numéricos, os indivíduos do gênero masculino Padrão Face Longa apresentaram disparidades em relação aos indivíduos Padrão I apenas nas grandezas relacionadas à altura facial, ao padrão de crescimento e à relação sagital (Tab. 6).

\section{DISCUSSÃO}

\section{Padrão de crescimento facial}

De acordo com a literatura, há uma clara evidência de que, na avaliação cefalométrica, a deformidade que caracteriza os indivíduos Padrão Face Longa reside predominantemente abaixo do plano palatino ${ }^{14}$. Esta afirmativa foi confirmada no presente estudo, onde os valores do ângulo do plano palatino não apresentaram diferenças significantes entre os indivíduos dos gêneros feminino e masculino, pertencentes ao Padrão I e Padrão Face Longa (Tab. 5, 6). Além disso, esta grandeza não demonstrou tendência ao dimorfismo sexual (Tab. $3,4)$. Por outro lado, as grandezas que descrevem as deformidades localizadas abaixo do plano palatino, tais como os ângulos do plano mandibular e goníaco foram diferentes entre o Padrão I e o Padrão Face Longa (Tab. 5, 6), corroborando os dados da literatura. Todavia, para esses ângulos, não ocorreu tendência ao dimorfismo sexual, isto é, a magnitude do crescimento vertical nos indivíduos do gênero feminino e seus efeitos foram semelhantes nos indivíduos do gênero masculino (Tab. 3, 4).

\section{Alturas faciais anteriores e posterior}

Altura facial anterior total (AFAT) e total perpendicular (AFATperp)

O uso da grandeza AFATperp tem a finalidade de reduzir a influência do erro geométrico na avaliação da AFAT, em indivíduos que apresentam displasias esqueléticas com conseqüências rotacionais na mandíbula ${ }^{11}$. Portanto, para os indivíduos Padrão Face Longa, o uso da AFATperp é mais adequado.

A presença de dimorfismo sexual foi observada nos indivíduos Padrão I. Essa diferença era esperada, uma vez que a altura facial anterior dos indivíduos masculinos é maior do que a dos indivíduos femininos em condições de normalidade ${ }^{25}$. Entre os indivíduos Padrão Face Longa essa diferença não foi detectada entre os gêneros, provavelmente porque a deformidade face longa foi severa o bastante para superar a menor altura facial esperada para as mulheres, tornando-a similar à dos homens. Essa evidência é destacada pela diferença estatisticamente significante existente entre as mulheres Padrão I e Padrão Face Longa, com relação aos valores da AFAT e AFATperp.

\section{Altura facial anterior média (AFAM)}

Esta grandeza foi significantemente diferente entre os indivíduos do gêneros feminino e masculino do grupo Padrão I, com as mulheres exibindo uma AFAM menor. Isto não ocorreu para os indivíduos do grupo Face Longa, o que novamente evidencia que as mulheres afetadas apresentam um aumento significativo para a altura da face média, capaz de anular a diferença esperada em relação ao gênero masculino. Isso contraria, embora de modo sutil, o conceito de que, cefalometricamente, a deformidade face longa localiza-se abaixo do plano palatino. 
Características cefalométricas do Padrão Face Longa: considerando o dimorfismo sexual

Tabela 3 - Comparação entre os gêneros feminino e masculino do grupo Padrão I.

\begin{tabular}{|c|c|c|c|c|c|c|c|}
\hline variáveis & unid. & médias (F) & d.p. & médias (M) & d.p. & $\mathbf{t}$ & p \\
\hline Co-Gn & $\mathrm{mm}$ & 119,86 & 5,10 & 134,15 & 3,20 & 9,203 & $<0,001^{*}$ \\
\hline Co-A & $\mathrm{mm}$ & 92,46 & 3,96 & 100,80 & 3,83 & 6,266 & $<0,001^{*}$ \\
\hline DifMxMd & $\mathrm{mm}$ & 27,40 & 4,20 & 33,35 & 3,21 & 4,484 & $<0,001^{*}$ \\
\hline AFAT & $\mathrm{mm}$ & 119,59 & 5,21 & 134,11 & 6,11 & 7,752 & $<0,001^{*}$ \\
\hline AFAl & $\mathrm{mm}$ & 66,70 & 5,00 & 77,02 & 4,87 & 6,125 & $<0,001^{*}$ \\
\hline AFAM & $\mathrm{mm}$ & 54,91 & 3,06 & 58,71 & 3,01 & 3,667 & $<0,001^{*}$ \\
\hline AFP & $\mathrm{mm}$ & 61,24 & 3,98 & 66,88 & 5,65 & 3,618 & $<0,001^{*}$ \\
\hline AFATperp & $\mathrm{mm}$ & 119,38 & 5,21 & 133,91 & 5,93 & 7,847 & $<0,001^{*}$ \\
\hline AFAlperp & $\mathrm{mm}$ & 65,21 & 4,74 & 75,76 & 4,23 & 6,779 & $<0,001^{*}$ \\
\hline $1-P P$ & $\mathrm{~mm}$ & 5,62 & 1,97 & 7,14 & 1,48 & 2,460 & $<0,05^{*}$ \\
\hline$\underline{6}-P P$ & $\mathrm{~mm}$ & 4,86 & 2,13 & 6,33 & 1,92 & 2,081 & $<0,05^{*}$ \\
\hline 1-PM & $\mathrm{mm}$ & 17,42 & 2,71 & 20,75 & 2,59 & 3,669 & $<0,001^{*}$ \\
\hline 6-PM & $\mathrm{mm}$ & 10,80 & 3,22 & 13,31 & 3,38 & 2,259 & $<0,05^{*}$ \\
\hline SNA & graus & 82,67 & 2,43 & 81,78 & 4,29 & 0,828 & $>0,05$ \\
\hline SNB & graus & 79,78 & 1,82 & 79,31 & 3,48 & 0,556 & $>0,05$ \\
\hline ANB & graus & 2,89 & 2,08 & 2,47 & 1,89 & 0,612 & $>0,05$ \\
\hline AnGon & graus & 120,09 & 4,45 & 123,23 & 6,29 & 1,807 & $>0,05$ \\
\hline AnPM & graus & 28,82 & 3,77 & 30,69 & 5,06 & 1,299 & $>0,05$ \\
\hline AnPP & graus & 9,88 & 2,87 & 7,95 & 3,01 & 1,945 & $>0,05$ \\
\hline 1.PP & graus & 113,57 & 6,49 & 115,88 & 7,42 & 0,999 & $>0,05$ \\
\hline IMPA & graus & 94,85 & 6,36 & 92,45 & 6,05 & 1,129 & $>0,05$ \\
\hline A1/3IF & graus & 80,01 & 4,23 & 76,17 & 4,26 & 2,663 & $<0,05^{*}$ \\
\hline NAP & graus & 4,39 & 4,51 & 4,10 & 2,33 & 0,220 & $>0,05$ \\
\hline $\begin{array}{l}\text { AFAlperp/ } \\
\text { AFATperp }\end{array}$ & - & 0,55 & 0,03 & 0,57 & 0,02 & 2,565 & $<0,05^{*}$ \\
\hline AFAI/AFAT & - & 0,56 & 0,03 & 0,57 & 0,02 & 1,791 & $>0,05$ \\
\hline AFAM/AFAI & - & 0,83 & 0,08 & 0,76 & 0,05 & 2,559 & $<0,05^{*}$ \\
\hline AFP/AFAT & - & 0,51 & 0,03 & 0,50 & 0,04 & 1,210 & $>0,05$ \\
\hline
\end{tabular}

* Diferença estatisticamente significante ao nível indicado.

Altura facial anterior inferior (AFAl) e inferior perpendicular (AFAlperp)

Analogamente à grandeza AFAT, a AFAI também pode sofrer influência da rotação mandibular, justificando-se o uso da grandeza AFAIperp para anulá-la.

O dimorfismo sexual esteve presente para AFAIperp para os indivíduos Padrão Face Longa e Padrão I. Esse dimorfismo foi resultado da interação entre a característica vinculada ao gênero masculino, que apresenta dimensão esquelética maior, e à deformidade esquelética, típica do $\mathrm{Pa}$ drão Face Longa.

Não houve dimorfismo sexual, entretanto, para o valor da AFAI em indivíduos Padrão Face Longa. Este resultado provavelmente é devido à retrusão mandibular comum nos indivíduos $\mathrm{Pa}$ drão Face Longa, mascarando a altura facial inferior real. Isso apenas comprova a inadequação dessa grandeza, que inviabiliza seu uso. 
Tabela 4 - Comparação entre os gêneros feminino e masculino do grupo Padrão Face Longa.

\begin{tabular}{|c|c|c|c|c|c|c|c|}
\hline variáveis & unid. & médias (F) & d.p. & médias (M) & d.p. & $\mathbf{t}$ & $\mathbf{p}$ \\
\hline Co-Gn & $\mathrm{mm}$ & 119,58 & 8,80 & 129,70 & 11,07 & 2,923 & $<0,01^{*}$ \\
\hline Co-A & $\mathrm{mm}$ & 89,80 & 5,15 & 94,48 & 6,15 & 2,368 & $<0,05^{*}$ \\
\hline DifMxMd & $\mathrm{mm}$ & 29,79 & 7,96 & 35,22 & 9,08 & 1,810 & $>0,05$ \\
\hline AFAT & $\mathrm{mm}$ & 133,31 & 7,82 & 139,62 & 12,70 & 1,796 & $>0,05$ \\
\hline AFAI & $\mathrm{mm}$ & 80,81 & 5,64 & 85,33 & 8,77 & 1,832 & $>0,05$ \\
\hline AFAM & $\mathrm{mm}$ & 56,40 & 3,24 & 57,02 & 5,25 & 0,426 & $>0,05$ \\
\hline AFP & $\mathrm{mm}$ & 57,19 & 5,22 & 62,62 & 6,07 & 2,736 & $<0,05^{*}$ \\
\hline AFATperp & $\mathrm{mm}$ & 131,71 & 7,63 & 138,36 & 12,64 & 1,919 & $>0,05$ \\
\hline AFAIperp & $\mathrm{mm}$ & 75,93 & 5,54 & 81,98 & 9,19 & 2,404 & $<0,05^{*}$ \\
\hline$\underline{1}-\mathrm{PP}$ & $\mathrm{mm}$ & 10,37 & 3,28 & 9,27 & 2,99 & 0,965 & $>0,05$ \\
\hline$\underline{6}-\mathrm{PP}$ & $\mathrm{mm}$ & 8,17 & 2,95 & 7,51 & 4,29 & 0,531 & $>0,05$ \\
\hline 1-PM & $\mathrm{mm}$ & 21,10 & 2,79 & 22,31 & 2,61 & 1,234 & $>0,05$ \\
\hline 6-PM & $\mathrm{mm}$ & 12,01 & 3,52 & 12,98 & 3,85 & 0,741 & $>0,05$ \\
\hline SNA & graus & 79,68 & 3,64 & 80,36 & 3,52 & 0,528 & $>0,05$ \\
\hline SNB & graus & 72,96 & 5,05 & 76,12 & 4,17 & 1,841 & $>0,05$ \\
\hline ANB & graus & 6,71 & 3,89 & 4,25 & 3,15 & 1,879 & $>0,05$ \\
\hline AnGon & graus & 131,32 & 5,71 & 132,09 & 6,36 & 0,357 & $>0,05$ \\
\hline AnPM & graus & 45,27 & 6,67 & 41,65 & 6,74 & 1,506 & $>0,05$ \\
\hline AnPP & graus & 9,33 & 4,36 & 7,72 & 2,82 & 1,149 & $>0,05$ \\
\hline 1.PP & graus & 109,67 & 8,72 & 114,87 & 6,12 & 1,831 & $>0,05$ \\
\hline IMPA & graus & 89,12 & 7,23 & 87,07 & 7,95 & 0,761 & $>0,05$ \\
\hline $\mathrm{A} 1 / 3 \mathrm{IF}$ & graus & 72,23 & 6,82 & 70,93 & 8,11 & 0,497 & $>0,05$ \\
\hline NAP & graus & 12,11 & 8,25 & 7,83 & 4,14 & 1,676 & $>0,05$ \\
\hline $\begin{array}{l}\text { AFAlperp/ } \\
\text { AFATperp }\end{array}$ & - & 0,58 & 0,02 & 0,59 & 0,02 & 2,498 & $<0,05^{*}$ \\
\hline AFAl/AFAT & - & 0,61 & 0,01 & 0,61 & 0,02 & 0,826 & $>0,05$ \\
\hline AFAM/AFAI & - & 0,70 & 0,03 & 0,67 & 0,06 & 1,792 & $>0,05$ \\
\hline AFP/AFAT & - & 0,43 & 0,04 & 0,45 & 0,04 & 1,535 & $>0,05$ \\
\hline
\end{tabular}

*Diferença estatisticamente significante ao nível de indicado.

Altura facial posterior (AFP)

A literatura é controversa em relação à grandeza da altura facial posterior. Autores têm demonstrado presença de altura facial posterior menor $^{6,9,19,21,23,26,31,34}$, maior ${ }^{7,17,18}$ ou ausência de diferença significante ${ }^{16,28,29,37}$ quando indivíduos Padrão I e Face Longa foram comparados. Os dados da literatura não fazem distinção para gênero. Neste estudo, os valores da altura facial posterior dos indivíduos Padrão Face Longa foram inferiores aos dos indivíduos Padrão I no gênero feminino, contribuindo, em associação com o incremento da AFAIperp, para gerar a característica do Padrão Face Longa.

Observou-se tendência ao dimorfismo sexual tanto para o grupo Padrão I como para o grupo Padrão Face Longa. Todavia, de acordo com os resultados obtidos, a altura facial posterior no gênero masculino não diferiu significativamente entre os grupos Padrão Face Longa e Padrão I, contrastan- 
Características cefalométricas do Padrão Face Longa: considerando o dimorfismo sexual

Tabela 5 - Comparação entre os indivíduos Padrão I e Padrão Face Longa do gênero feminino

\begin{tabular}{|c|c|c|c|c|c|c|c|}
\hline variáveis & unid. & médias (PI) & d.p. & médias (PFL) & d.p. & $t$ & $\mathrm{p}$ \\
\hline Co-Gn & $\mathrm{mm}$ & 119,86 & 5,10 & 119,58 & 8,80 & 0,137 & $>0,05$ \\
\hline Co-A & $\mathrm{mm}$ & 92,46 & 3,96 & 89,80 & 5,15 & 2,024 & $<0,05^{*}$ \\
\hline DifMxMd & $\mathrm{mm}$ & 27,40 & 4,20 & 29,79 & 7,96 & 1,326 & $>0,05$ \\
\hline AFAT & $\mathrm{mm}$ & 119,59 & 5,21 & 133,31 & 7,82 & 7,254 & $<0,001^{*}$ \\
\hline AFAI & $\mathrm{mm}$ & 66,70 & 5,00 & 80,81 & 5,64 & 9,187 & $<0,001^{*}$ \\
\hline AFAM & $\mathrm{mm}$ & 54,91 & 3,06 & 56,40 & 3,24 & 1,634 & $>0,05$ \\
\hline AFP & $\mathrm{mm}$ & 61,24 & 3,98 & 57,19 & 5,22 & 3,047 & $<0,01^{*}$ \\
\hline AFATperp & $\mathrm{mm}$ & 119,38 & 5,21 & 131,71 & 7,63 & 6,620 & $<0,001^{*}$ \\
\hline AFAlperp & $\mathrm{mm}$ & 65,21 & 4,74 & 75,93 & 5,54 & 7,229 & $<0,001^{*}$ \\
\hline $1-P P$ & $\mathrm{~mm}$ & 5,62 & 1,97 & 10,37 & 3,28 & 6,199 & $<0,001^{*}$ \\
\hline$\underline{6}-P P$ & $\mathrm{~mm}$ & 4,86 & 2,13 & 8,17 & 2,95 & 4,498 & $<0,001^{*}$ \\
\hline 1-PM & $\mathrm{mm}$ & 17,42 & 2,71 & 21,10 & 2,79 & 4,630 & $<0,001^{*}$ \\
\hline 6-PM & $\mathrm{mm}$ & 10,80 & 3,22 & 12,01 & 3,52 & 1,242 & $>0,05$ \\
\hline SNA & graus & 82,67 & 2,43 & 79,68 & 3,64 & 3,394 & $<0,01^{*}$ \\
\hline SNB & graus & 79,78 & 1,82 & 72,96 & 5,05 & 6,416 & $<0,001^{*}$ \\
\hline ANB & graus & 2,89 & 2,08 & 6,71 & 3,89 & 4,338 & $<0,001^{*}$ \\
\hline AnGon & graus & 120,09 & 4,45 & 131,32 & 5,71 & 7,660 & $<0,001^{*}$ \\
\hline $\mathrm{AnPM}$ & graus & 28,82 & 3,77 & 45,27 & 6,67 & 10,716 & $<0,001^{*}$ \\
\hline AnPP & graus & 9,88 & 2,87 & 9,33 & 4,36 & 0,520 & $>0,05$ \\
\hline 1.PP & graus & 113,57 & 6,49 & 109,67 & 8,72 & 1,773 & $>0,05$ \\
\hline IMPA & graus & 94,85 & 6,36 & 89,12 & 7,23 & 2,923 & $<0,01^{*}$ \\
\hline $\mathrm{A} 1 / 3 \mathrm{IF}$ & graus & 80,01 & 4,23 & 72,23 & 6,82 & 4,820 & $<0,001^{*}$ \\
\hline NAP & graus & 4,39 & 4,51 & 12,11 & 8,25 & 4,105 & $<0,001^{*}$ \\
\hline $\begin{array}{l}\text { AFAlperp/ } \\
\text { AFATperp }\end{array}$ & - & 0,55 & 0,03 & 0,58 & 0,02 & 4,805 & $<0,001^{*}$ \\
\hline AFAl/AFAT & - & 0,56 & 0,03 & 0,61 & 0,01 & 7,703 & $<0,001^{*}$ \\
\hline AFAM/AFAI & - & 0,83 & 0,08 & 0,70 & 0,03 & 6,844 & $<0,001^{*}$ \\
\hline AFP/AFAT & - & 0,51 & 0,03 & 0,43 & 0,04 & 8,305 & $<0,001^{*}$ \\
\hline
\end{tabular}

*Diferença estatisticamente significante ao nível indicado.

do com a altura facial posterior no gênero feminino, que foi significantemente diferente entre os grupos estudados. Clinicamente, a menor dimensão da altura facial posterior encontrada no gênero feminino contribui para gerar uma face longa mais severa nesses indivíduos, quando comparados aos do gênero masculino.

\section{Proporções entre as alturas faciais}

É essencial, além de observar as alterações ocorridas nas grandezas cefalométricas relacionadas às alturas faciais, considerar também as proporções existentes entre as várias dimensões da face. A estética nos indivíduos Padrão Face Longa é afetada, principalmente, devido a uma alteração desproporcional dessas dimensões. No presente estudo, houve dimorfismo sexual nos indivíduos Padrão Face Longa somente na proporção entre AFATperp e AFAIperp, diferentemente dos indivíduos Padrão I, que têm também 
Tabela 6 - Comparação entre os indivíduos Padrão I e Padrão Face Longa do gênero masculino.

\begin{tabular}{|c|c|c|c|c|c|c|c|}
\hline variáveis & unid. & médias (PI) & d.p. & médias (PFL) & d.p. & $\mathrm{t}$ & $p$ \\
\hline Co-Gn & $\mathrm{mm}$ & 134,15 & 3,20 & 129,70 & 11,07 & 1,39 & $>0,05$ \\
\hline Co-A & $\mathrm{mm}$ & 100,80 & 3,83 & 94,45 & 6,15 & 3,111 & $<0,01^{*}$ \\
\hline DifMxMd & $\mathrm{mm}$ & 33,35 & 3,21 & 35,22 & 9,08 & 0,696 & $>0,05$ \\
\hline AFAT & $\mathrm{mm}$ & 134,11 & 6,11 & 139,62 & 12,70 & 1,398 & $>0,05$ \\
\hline AFAl & $\mathrm{mm}$ & 77,02 & 4,87 & 85,33 & 8,77 & 2,959 & $<0,01^{*}$ \\
\hline AFAM & $\mathrm{mm}$ & 58,71 & 3,01 & 57,02 & 5,25 & 0,993 & $>0,05$ \\
\hline AFP & $\mathrm{mm}$ & 66,88 & 5,65 & 62,62 & 6,07 & 1,816 & $>0,05$ \\
\hline AFATperp & $\mathrm{mm}$ & 133,91 & 5,93 & 138,36 & 12,64 & 1,142 & $>0,05$ \\
\hline AFAlperp & $\mathrm{mm}$ & 75,76 & 4,23 & 81,98 & 9,19 & 2,203 & $<0,05^{*}$ \\
\hline $1-P P$ & $\mathrm{~mm}$ & 7,14 & 1,48 & 9,27 & 2,99 & 2,282 & $<0,05^{*}$ \\
\hline$\underline{6}-\mathrm{PP}$ & $\mathrm{mm}$ & 6,33 & 1,92 & 7,51 & 4,29 & 0,904 & $>0,05$ \\
\hline 1-PM & $\mathrm{mm}$ & 20,75 & 2,59 & 22,31 & 2,61 & 1,503 & $>0,05$ \\
\hline 6-PM & $\mathrm{mm}$ & 13,31 & 3,38 & 12,98 & 3,85 & 0,231 & $>0,05$ \\
\hline SNA & graus & 81,78 & 4,29 & 80,36 & 3,52 & 0,902 & $>0,05$ \\
\hline SNB & graus & 79,31 & 3,48 & 76,12 & 4,17 & 2,089 & $<0,05^{*}$ \\
\hline ANB & graus & 2,47 & 1,89 & 4,25 & 3,15 & 1,727 & $>0,05$ \\
\hline AnGon & graus & 123,23 & 6,29 & 132,09 & 6,36 & 3,499 & $<0,01^{*}$ \\
\hline AnPM & graus & 33,69 & 5,06 & 41,65 & 6,74 & 4,619 & $<0,001^{*}$ \\
\hline AnPP & graus & 7,95 & 3,01 & 7,72 & 2,82 & 0,194 & $>0,05$ \\
\hline 1.PP & graus & 115,88 & 7,42 & 114,87 & 6,12 & 0,367 & $>0,05$ \\
\hline IMPA & graus & 92,45 & 6,05 & 87,07 & 7,95 & 1,911 & $>0,05$ \\
\hline $\mathrm{A} 1 / 3 \mathrm{IF}$ & graus & 76,17 & 4,26 & 70,93 & 8,11 & 2,044 & $>0,05$ \\
\hline NAP & graus & 4,10 & 3,33 & 7,83 & 4,14 & 2,809 & $<0,01^{*}$ \\
\hline $\begin{array}{l}\text { AFAlperp/ } \\
\text { AFATperp }\end{array}$ & - & 0,57 & 0,02 & 0,59 & 0,02 & 3,599 & $<0,01^{*}$ \\
\hline AFAI/AFAT & - & 0,57 & 0,02 & 0,61 & 0,02 & 4,486 & $<0,001^{*}$ \\
\hline AFAM/AFAI & - & 0,76 & 0,05 & 0,67 & 0,05 & 4,229 & $<0,001^{*}$ \\
\hline AFP/AFAT & - & 0,50 & 0,04 & 0,45 & 0,04 & 2,996 & $<0,01^{*}$ \\
\hline
\end{tabular}

*Diferença estatisticamente significante ao nível indicado.

a proporção AFAT e AFAI diferente entre homens e mulheres.

\section{Relação maxilomandibular e os comprimen- tos efetivos da maxila e da mandíbula}

A posição da maxila, avaliada pelo ângulo SNA, foi de retrusão em relação à base do crânio nos indivíduos Padrão Face Longa. Esse seria um evento esperado para os portadores da deformidade avaliada ${ }^{21,23}$, mas foi significante apenas no gê- nero feminino. A retrusão maxilar provavelmente está associada a uma redução no comprimento efetivo da maxila (Co-A), conforme foi constatado no presente estudo. Ao mesmo tempo, isso também pode ser resultado da redução do comprimento da maxila (ENA-ENP), observada por Haralabakis et al. ${ }^{21}$ Todavia, esse resultado diverge das observações da literatura ${ }^{2,17,18,35}$, onde não foram encontradas diferenças significantes quanto ao valor desse ângulo. Para o ângulo SNA, não se 
observou tendência ao dimorfismo sexual, apesar de existir diferença significante entre as mulheres Padrão Face Longa e Padrão I.

No que se refere à retrusão mandibular, não foi observada presença de dimorfismo sexual na comparação entre os grupos Padrão I e Padrão Face Longa. Quando os gêneros feminino e masculino foram avaliados isoladamente, ambos apresentaram retrusão significante para os indivíduos Padrão Face Longa, sendo esta mais acentuada para o gênero feminino, assim como a retrusão maxilar. Isso permite especular que existe uma maior tendência retrognata para o gênero feminino, corroborando os achados de Behrents ${ }^{4}$. Não obstante, Haralabakis et al. ${ }^{21}$ encontraram um comprimento do corpo mandibular menor para o gênero feminino, provavelmente correlacionado a uma altura do ramo diminuída.

A avaliação da relação maxilomandibular, baseada no valor do ângulo ANB, mostrou um degrau positivo mais acentuado nos indivíduos Padrão Face Longa. Novamente, os resultados obtidos neste trabalho mantêm coerência na avaliação da relação maxilomandibular, não ocorrendo dimorfismo sexual para os grupos Padrão I e Padrão Face Longa. No entanto, no gênero feminino, os indivíduos do grupo Padrão Face Longa exibiram maior degrau positivo, em comparação com o masculino, atestando, dessa forma, uma característica mais retrognata dessa amostra. Simultaneamente, uma maxila mais retruída foi observada no gênero feminino do grupo Padrão Face Longa, assim como uma mandíbula suficientemente retruída para aumentar o valor do ângulo ANB. Em síntese, parece que o quadro final da retrusão maxilomandibular, observado no gênero feminino do grupo Padrão Face Longa, é resultante do somatório de retrusão maxilar e retrusão mandibular, definido pelo padrão do indivíduo, semelhante aos achados de Isaacson et al. ${ }^{23}$

A retrusão mandibular foi enfatizada com a avaliação dos ângulos do terço inferior da face e NAP. Dimorfismo sexual não foi constatado para os indivíduos Padrão Face Longa, ocorrendo apenas para o ângulo do terço inferior da face (Al/31F) nos indivíduos Padrão I. Como era lícito esperar, o ângulo de convexidade facial (NAP) foi maior para ambos os gêneros no grupo Padrão Face Longa. $\mathrm{O}$ ângulo do terço inferior da face evidenciou diferença apenas para os indivíduos do gênero feminino, confirmando o maior comprometimento dessa variável nesses indivíduos.

\section{Relações dentárias com as suas bases apicais}

Quando avaliado o grupo Padrão I, houve presença de dimorfismo sexual em relação às distâncias ortogonais dos incisivos superiores e inferiores, porém, esse quadro desapareceu quando os indivíduos do Padrão Face Longa foram comparados. Isso confirma a existência de compensação dentária nos portadores de Padrão Face Longa, em maior intensidade no gênero feminino, já que a diferença observada entre os indivíduos Padrão I e Padrão Face Longa do gênero masculino foi muito pequena. A exceção é a distância dos molares inferiores ao plano mandibular, que nunca foi afetada pela doença. Esse resultado corrobora a tese de maior gravidade da discrepância face longa no gênero feminino.

\section{CONCLUSÕES}

Com os resultados obtidos neste trabalho, onde se procurou definir a presença de dimorfismo sexual em portadores de más oclusões do Padrão Face Longa, por meio da cefalometria, parece razoável concluir que:

1) Houve predominância de similaridade para grandezas lineares e angulares dos indivíduos do gêneros feminino e masculino Padrão Face Longa, revelando que a deformidade foi mais grave no gênero feminino, sendo severa o bastante para superar o menor tamanho esquelético esperado para esse grupo;

2) Quando os indivíduos do gênero feminino Padrão I e Padrão Face Longa foram comparados, evidenciou-se diferenças estatisticamente signifi- 
cantes para a grande maioria dos valores médios das grandezas, lineares ou angulares, que foram maiores no Padrão Face Longa.

3) Os indivíduos do gênero masculino Padrão Face Longa apresentaram disparidades em relação aos indivíduos Padrão I apenas nas grandezas relacionadas à altura facial, ao padrão de crescimento e à relação sagital.

\section{AGRADECIMENTOS}

À empresa Radiomemory, a qual nos forneceu o software Radiocef 2.0, pelo constante suporte técnico fornecido durante a realização do estudo.

\title{
Cephalometric features of Long Face Pattern: concerns about sexual dimorphism
}

\begin{abstract}
Aim: The purpose of this study was to evaluate the presence of different cephalometric characteristics between genders with Long Face Pattern. Methods: a total of 73 lateral cephalometric radiographs were evaluated, with 34 Long Face Pattern and 39 Class I Pattern (control) subjects, sampled with basis on the facial morphology, regardless the sagital occlusal relationships. Facial growth pattern, anterior and posterior facial heights, inter-jaw relationship and dentoskeletal relationship were evaluated. Results: It was observed sexual dimorphism in Class I Pattern subjects $(p<0.001)$ in the effective length of maxilla and mandible, as well as the facial heights. Also, significant differences were observed among facial height ratios and the distances from incisors and molars to palatal and mandibular plane. For Long Face Pattern subjects, these cephalometric variables showed no sexual dimorphism ( $p>0.05)$, except the effective length of maxilla and mandible, lower anterior and posterior facial heights $(p<0.05)$. Conclusions: It was concluded that sexual dimorphism was evident in Class I Pattern subjects due to the skeletal size differences. However, in Long Face Pattern subjects, similarity was observed between genders, showing that the skeletal deformity was more severe in female subjects, enough to achieve skeletal size comparable to those of male subjects.
\end{abstract}

Key words: Cephalometry. Deformities. Dimorphism. Long Face Pattern.

\section{REFERÊNCIAS}

1. AN, T. L.; CUOGHI, O. A. O uso da estatística na Ortodontia. R Dental Press Ortodon Ortop Maxilar, Maringá, v. 9, n.6, p. 97-108, nov./dez. 2004

2. ANGELILLO, J. C.; DOLAN, E. A. The surgical correction of vertical maxillary excess: long face syndrome. Ann Plast Surg, Boston, v. 8, p. 64-70, 1982.

3. ARVYSTAS, M. G. Treatment of anterior skeletal open-bite deformity. Am J Orthod, St. Louis, v. 72, p. 147-164, 1977.

4. BEHRENTS, R. G. JCO interviews Dr. Rolf Behrens on adult craniofacial growth. J Clin Orthod, Boulder, v. 20, p. 842-847, 1986.

5. BELL, W. H. Correction of skeletal type of anterior open bite. J Oral Surgery, Chicago, v. 29, p. 706-714, 1971.

6. BELL, W. H.; CREEKMORE, T. D.; ALEXANDER, R. G. Surgical correction of the long face syndrome. Am J Orthod, St. Louis, v. 71, p. 40-67, 1977.

7. BJÖRK, A. Prediction of mandibular growth rotation. Am J Orthod, St. Louis, v. 55, p. 585-599, 1969.

8. BLANCHETTE, M. E.; NANDA, R. S.; CURRIER, G. F.; GHOSH, J.; NANDA, S. K. A longitudinal cephalometric study of the soft tissue profile of short-and long-face syndromes from 7 to 17 years. Am J Orthod Dentofacial Orthop, St. Louis, v. 109, p. 116-131, 1996.
9. CANGIALOSI, T. I. Skeletal morphologic features of anterior open bite. Am J Orthod Dentofacial Orthop, St. Louis, v. 85, p. 28-36, 1984.

10. CAPELOZZA FILHO, L.; SOUZA, S. L. M. C.; CAVASSAN, A. O.; OZAWA, T. O. A altura facial anterior inferior nas más oclusões do Padrão II, deficiência mandibular. R Dental Press Ortodon Ortop Facial, Maringá, v. 9, p. 39-47, 2004.

11. CAPELOZZA FILHO, L. Diagnóstico em Ortodontia. Maringá: Dental Press, 2004.

12. CARDOSO, M. A.; BERTOZ, F. A.; REIS, S. A. B.; CAPELOZZA FILHO, L. Estudo das características oclusais em portadores de padrão face longa com indicação de tratamento ortodônticocirúrgico. R Dental Press Ortodon Ortop Maxilar, Maringá, v. 7, n. 6, p. 63-70, nov. dez. 2002.

13. CARDOSO, M. A. Estudo das características cefalométricas do padrão face longa. 2003. 163 f. Dissertação (Mestrado em Ortodontia)-Faculdade de Odontologia de Araçatuba, Universidade Estadual Paulista, Araçatuba, 2003.

14. CARDOSO, M. A.; BERTOZ, F. A.; CAPELOZZA FILHO, L.; REIS, S. A. B. Características cefalométricas do padrão face longa. R Dental Press Ortodon Ortop Maxilar, Maringá, v. 10, n. 2, p. 29-43, mar./abr. 2005. 
15. EPKER, B. N. Superior surgical repositioning of the maxilla: long term results. J Maxillofac Sur, Stuttgart, v. 9, p. 237-246, 1981

16. FIELDS, H. W.; PROFFIT, W. R.; NIXON, W. L.; PHILLIPS, C.; STANEK, E. Facial pattern differences in long-faced children and adults. Am J Orthod, St. Louis, v. 85, p. 217-223, 1984.

17. FISH, L. C.; WOLFORD, L. M.; EPKER, B. N. Surgical-orthodontic correction of vertical maxillary excess. Am J Orthod, St. Louis, v. 73, p. 241-257, 1978.

18. FITZPATRICK, B. N. The long face and V. M. E. Aust Orthod, Brisbane, v. 8, p. 82-89, 1984

19. FROST, D. E.; FONSECA, R. J.; TURVEY, T. A.; HALL, D. J. Cephalometric diagnosis and surgical-orthodontic correction of apertognathia. Am J Orthod, St. Louis, v. 78, p. 657-669, 1980.

20. GALLANGHER, D. M.; BELL, W. H.; STORUM, K. A. Soft tissue changes associated with advancement genioplasty performed concomitantly with superior repositioning of the maxilla. J Oral Maxillofac Surg, Philadelphia, v. 42, p. 238-242, 1984

21. HARALABAKIS, S.; YAGTZIS, S. C.; TOUTOUNTZAKIS, N. M Cephalometric characteristics of open bite in adults: a three dimensional cephalometric evaluation. Int J Adult Orthodon Orthognath Surg, Chicago, v. 9, p. 222-232, 1994.

22. HOUSTON, W. J. The analysis of errors in orthodontic measurements. Am J Orthod, St. Louis, v. 83, p. 382-390, 1983

23. ISAACSON, J. R.; ISAACSON, R. J.; SPEIDEL, T. M.; WORMS F. W. Extreme variations in vertical facial growth and associated variation in skeletal and dental relations. Angle Orthod, Appleton, v. 41, p. 219-229, 1971.

24. MOLONEY, F.; WEST, R. A.; McNEILL, W. Surgical correction of vertical maxillary excess: a re-evaluation. J Maxillofac Surg, Edinburgh, v. 10, n.2, p. 84-91, 1982.

25. NAHOUM, H. I. Vertical proportions and the palatal plane in anterior open-bite. Am J Orthod, St. Louis, v. 59, p. 273-282, 1971.

26. NAHOUM, H. I.; HOROWITZ, S. L.; BENEDICTO, E. A. Varieties of anterior open-bite. Am J Orthod, St. Louis, v. 61, p. 486-492, 1972.

27. NAHOUM, H. I. Anterior open-bite: a cephalometric analysis and suggested treatment procedures. Am J Orthod, St. Louis, v. 67, p. $513-521,1975$.

28. NANDA, S. K. Patterns of vertical growth in the face. Am J Orthod Dentofacial Orthop, St. Louis, v. 93, p.103-116, 1988.
29. NANDA, S. K. Growth patterns in subjects with long and short faces. Am J Orthod Dentofacial Orthop, St. Louis, v. 98, p. 247-258, 1990

30. OPDEBEECK, H.; BELL, W. H.; EISENFELD, J.; MISHELEVIC, D. Comparative study between the SFS and LFS rotation as a possible morphogenic mechanism. Am J Orthod, St. Louis, v. 74 p. 509-521, 1978

31. PRITTINEN, J. R. Orthodontic diagnosis of long face syndrome. Gen Dent, Chicago, v. 44, p. 348-351, 1996.

32. PRITTINEN, J. R. Orthodontic management of long face syndrome. Gen Dent, Chicago, v. 45, p. 568-572, 1997

33. REIS, S A B. CAPELOZZA FILHO L: CARDOSO, M A SCANAVINI, M. A. Características cefalométricas dos indivíduos Padrão I. R Dental Press Ortodon Ortop Maxilar, Maringá v. 10, no. 1, p. 67-78, 2005.

34. SASSOUNI, V.; NANDA, S. Analysis of dentofacial vertical proportions. Am J Orthod, St. Louis, v. 50, p. 801-823, 1964.

35. SCHENDEL, S. A.; EISENFELD, J.; BELL, W. H.; EPKER, B. N.; MISHELEVICH, D. J. The long face syndrome: vertical maxillary excess. Am J Orthod, St. Louis, v. 70, p. 398-408, 1976.

36. SCHENDEL, S. A.; CARLOTTI JR., A. E. Variations of total vertical maxillary excess. J Oral Maxillofac Surg, Philadelphia, v. 43, p. 590-596, 1985.

37. SUBTELNY, J. D.; SAKUDA, M. Open-bite: diagnosis and treatment. Am J Orthod, St. Louis, v. 50, p. 337-358, 1964

38. VAN DER BEEK, M. C. J.; HOEKSMA, J. B.; ANDERSEN, B. P. Vertical facial growth: a longitudinal study from 7 to 14 year of age. Eur J Orthod, Oxford, v. 13, p. 202-228, 1991.

39. LINDEN, P. G. M. van der. O desenvolvimento das faces longas e curtas e as limitações do tratamento. R Dental Press Ortodon Ortop Facial, Maringá, v. 4, n. 6, p. 6-11, nov./dez. 1999.

40. VASCONCELOS, M. H. F. Avaliação de um programa de traçado cefalométrico. 2000. 178 f. Tese (Doutorado em Ortodontia)-Faculdade de Odontologia de Bauru, Universidade de São Paulo, Bauru, 2000.

41. VIG, K. W.; TURVEY, T. A. Surgical correction of vertical maxillary excess during adolescence. Int J Adult Orthodon Orthognath Surg, Chicago, v. 4, p. 119-128, 1989.

42. WOLFORD, L. M.; HILLIARD, F. W. The surgical-orthodontic correction of vertical dentofacial deformities. J Oral Surg, Chicago, v. 39, p. 883-897, 1981.
Endereço para correspondência

Leopoldino Capelozza Filho

Rua Servio Tulio Coube, 2-70 - Bairro Infante Dom Henrique

CEP: 17.012632 - Bauru/SP

E-mail: Icapelozza@yahoo.com.br 